\title{
A SHARPENING OF A PROBLEM ON BERNSTEIN POLYNOMIALS AND CONVEX FUNCTIONS AND RELATED RESULTS
}

\author{
ANDRZEJ KOMISARSKI AND TERESA RAJBA
}

Abstract. We present a short proof of a conjecture proposed by I. Raşa (2017), which is an inequality involving basic Bernstein polynomials and convex functions. This proof was given in the letter to I. Raşa (2017). The methods of our proof allow us to obtain some extended versions of this inequality as well as other inequalities given by I. Raşa. As a tool we use stochastic convex ordering relations. We propose also some generalizations of the binomial convex concentration inequality. We use it to insert some additional expressions between left and right sides of the Raşa inequalities.

Mathematics subject classification (2010): 60E15, 39B62.

Keywords and phrases: Bernstein polynomials, stochastic convex ordering, convex functions, functional inequalities including convexity.

\section{REFERENCES}

[1] U. ABEL, An inequality involving Bernstein polynomials and convex functions, J. Approx. Theory 222 (2017), 1-7.

[2] U. ABEL, I. RAŞA, A sharpening problem on Bernstein polynomials and convex functions, arXiv preprint, arXiv:1707.00127v1 [math.CA] 1 Jul 2017.

[3] F. Altomare, M. Cappelletti Montano, V. Leoness a, I. Raşa, Markov Operators, Positive Semigroups and Approximation Processes, de Gruyter Studies in Mathematics 61, Walter de Gruyter GmbH, Berlin/Boston, 2014.

[4] F. Altomare, M. Cappelletti Montano, V. Leonessa, I. Raşa, A generalization of Kantorovich operators for convex compact subsets, Banach J. Math. Anal. 11 (2017), 591-614.

[5] G. Hardy, J. E. Littlewood, G. Pólya, Inequalities, Second Edition, Cambridge University Press, 1952.

[6] A. Komisarski, T. RajBa, Muirhead inequality for convex orders and a problem of I. Raşa on Bernstein polynomials, J. Math. Anal. Appl. 458 (2018), 821-830.

[7] A. Komisarski, T. RAJBA, Letter to Ioan Raşa, 2017,

[8] G. G. LoRentz, Bernstein polynomials, Mathematical Expositions, No. 8, University of Toronto Press, Toronto 1953.

[9] A. Marshall, I. Olkin, B. Arnold, Inequalities: Theory of Majorization and Its Applications, Second Edition, Springer, 2011.

[10] J. MrowiEC, T. RAJBA, S. WA̧SOWICZ, A solution to the problem of Raşa connected with Bernstein polynomials, J. Math. Anal. Appl. 446 (2017), 864-878.

[11] J. OHLIN, On a class of measures of dispersion with application to optimal reinsurance, ASTIN Bulletin 5 (1969), 249-266.

[12] I. RAŞA, 2. Problem, p. 164. In: Report of Meeting Conference on Ulam's Type Stability, Rytro, Poland, June 2-6, 2014, Ann. Univ. Paedagog. Crac. Stud. Math. 13 (2014), 139-169, doi: 10.2478/aupcsm-2014-0011.

[13] I. RAŞA, Bernstein polynomials and convexity: recent probabilistic and analytic proofs, The Workshop "Numerical Analysis, Approximation and Modeling", T. Popoviciu Institute of Numerical Analysis, Cluj-Napoca, June 14, 2017, http://ictp.acad.ro/zileleacademice-clujene-2017/.

[14] M. Shaked, J. G. Shanthikumar, Stochastic orders, Springer, 2007. 